moderately obese subjects in our study was identical to that reported in a recent meta-analysis on long-term pharmacotherapy for obesity. ${ }^{2}$

In response to Ornish's letter: the low-carbohydrate diet was based on Atkins's book, although we encouraged the study participants to consume a variety of protein and fat sources to maintain successful long-term adherence. We specifically reported on retention in detail and noted that adherence was higher in this trial than in any other trial of a similar length that we know of. The setting and methods we used to maximize adherence rates permitted a robust test of the three dietary strategies, as assessed by weight loss and established measurements of lipids, glycemic control, and hepatic and inflammatory biomarkers. As noted by Manzoni and colleagues, we did not assess cost-effectiveness. However, we believe that cost-effective interventions can be implemented in the workplace, with the use of group meetings and by working with food-service providers.
Several readers have informed us that our online Supplementary Appendix was incomplete. We have updated the appendix to provide more complete information.

Iris Shai, R.D., Ph.D.

Ben-Gurion University

Beer-Sheva 84105, Israel

irish@bgu.ac.il

Yaakov Henkin, M.D.

Soroka University Medical Center

Beer-Sheva 84101, Israel

Meir J. Stampfer, M.D., Dr.P.H.

Harvard Medical School

Boston, MA 02115

1. Shai I, Vardi H, Shahar DR, et al. Adaptation of international nutrition databases and data-entry system tools to a specific population. Public Health Nutr 2003;6:401-6.

2. Rucker D, Padwal R, Li SK, Curioni C, Lau DC. Long term pharmacotherapy for obesity and overweight: updated metaanalysis. BMJ 2007;335:1194-9.

\title{
Tibolone in Older Postmenopausal Women
}

TO THE EDITOR: In a randomized study of the effect of tibolone on fracture rates among postmenopausal women between the ages of 60 and 85 years, Cummings et al. (Aug. 14 issue) ${ }^{1}$ report that tibolone significantly reduced the risk of invasive breast cancer. The authors also report that this finding contradicted the results of the observational Million Women Study, which showed that the use of tibolone for up to 5 years was associated with an increased risk of breast cancer. ${ }^{2}$ In analyses combining clinical-trial and observational-study cohorts, the Women's Health Initiative (WHI) showed that a decreased risk of breast cancer was associated with estrogen therapy only if estrogen was given several years after menopause and not when it was begun soon thereafter. ${ }^{3}$

Since women in nontrial settings usually begin such hormonal therapy shortly after the onset of menopause, the gap time after menopause provides a potential effect modifier, suggesting caution in extrapolating findings to women closer to menopause. In the WHI randomized trial, estrogen plus progestin increased the risk of breast cancer that was diagnosed at an advanced stage, suggesting diagnostic delay with fewer cancers in the hormone group through the first 3 years of follow-up. ${ }^{4}$ The current tibolone trial was reported after a median of 34 months and without information on tumor stage. Although tibolone does not increase breast density, as does combined hormone therapy, ${ }^{5}$ could diagnostic delay account for some of the breast-cancer findings?

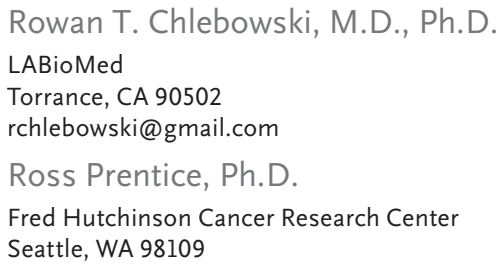

Dr. Chlebowski reports receiving consulting fees from AstraZeneca, Novartis, Eli Lilly, and Wyeth and grant support from Eli Lilly, Organon, and Amgen. No other potential conflict of interest relevant to this letter was reported.

1. Cummings SR, Ettinger B, Delmas PD, et al. The effects of tibolone in older postmenopausal women. N Engl J Med 2008; 359:697-708.

2. Beral V, Million Women Study Collaborators. Breast cancer and hormone-replacement therapy in the Million Women Study. Lancet 2003;362:419-27. [Erratum, Lancet 2003;362:1160.]

3. Prentice RL, Chlebowski RT, Stefanick ML, et al. Conjugated equine estrogens and breast cancer risk in the Women's Health Initiative clinical trial and observational study. Am J Epidemiol 2008;167:1407-15. 
4. Chlebowski RT, Anderson GL, Pettinger M, et al. Estrogen plus progestin and breast cancer detection by means of mammography and breast biopsy. Arch Intern Med 2008;168:370-7.

5. Lundström E, Christow A, Kersemaekers W, et al. Effects of tibolone and continuous combined hormone replacement therapy on mammographic breast density. Am J Obstet Gynecol 2002; 186:717-22.

TO THE EDITOR: With respect to the study by Cummings et al, we raise two issues: First, among the risk factors for stroke, the authors did not include the presence of high triglyceride levels and low levels of high-density lipoprotein (HDL) cholesterol, which have been shown to be related to ischemic cerebrovascular events. ${ }^{1,2}$ Were these values similar in the two study groups at baseline? Were they modified by the administration of tibolone?

Second, regarding gynecologic outcomes, patients who were treated with tibolone had a significantly higher incidence of mild cervical dysplasia on cytologic analysis than did those receiving placebo ( $7.6 \%$ vs. $3.2 \%, \mathrm{P}=0.009)$, but there were no differences between the groups in the incidence of moderate-to-severe dysplasia. Several studies have noted a synergistic role of sex hormones with human papillomavirus (HPV) infection in facilitating cervical carcinogenesis by inducing squamous metaplasia and by modifying the local immune microenvironment. ${ }^{3,4}$ This carcinogenic process requires several years. In the study by Cummings et al., the median duration of treatment was 34 months. We wonder whether in this period of time the possible HPV hyperactivation induced by tibolone could have caused mild dysplasia, whereas higher-grade dysplasia would have required a longer exposure.

Maurizio Serati, M.D.

Stefano Uccella, M.D.

PierFrancesco Bolis, M.D.

University of Insubria

21100 Varese, Italy

mauserati@hotmail.com

1. Koren-Morag N, Tanne D, Graff E, Goldbourt U. Low- and high-density lipoprotein cholesterol and ischemic cerebrovascular disease: the bezafibrate infarction prevention registry. Arch Intern Med 2002;162:993-9.

2. Lindenstrom E, Boysen G, Nyboe J. Influence of total cholesterol, high density lipoprotein cholesterol, and triglycerides on risk of cerebrovascular disease: the Copenhagen City Heart Study. BMJ 1994;309:11-5. [Erratum, BMJ 1994;309:1619.]

3. Nair HB, Luthra R, Kirma N, et al. Induction of aromatase expression in cervical carcinomas: effects of endogenous estro- gen on cervical cancer cell proliferation. Cancer Res 2005;65: 11164-73.

4. Delvenne P, Herman L, Kholod N, et al. Role of hormone cofactors in the human papillomavirus-induced carcinogenesis of the uterine cervix. Mol Cell Endocrinol 2007;264:1-5.

the aUthors Reply: Chlebowski and Prentice wonder whether a delay in diagnosis might account for some of the reduction in the risk of breast cancer that we observed among patients receiving tibolone. There was no significant difference between the groups in the use of mammography. As Chlebowski and Prentice note, tibolone does not appear to increase breast density, and there is no other reason why tumors would be more difficult to detect in women taking tibolone. Although data about the stage of cancers at diagnosis were not available in the trial, it seems unlikely that diagnostic delay could account for a meaningful part of the $68 \%$ decreased risk of invasive breast cancer.

Serati and colleagues ask whether changes in cholesterol and triglycerides might account for the increased risk of stroke we observed in patients receiving tibolone. We did not measure lipid concentrations in our trial, precluding an analysis of how much of the increased risk of stroke might have been due to changes in lipids. However, in previous placebo-controlled trials, ${ }^{1}$ 2 years of treatment with $1.25 \mathrm{mg}$ or $2.5 \mathrm{mg}$ of tibolone daily resulted in decreases in HDL cholesterol levels of $14 \%$ and $22 \%$, decreases in triglyceride levels of $21 \%$ and $23 \%$, and decreases in lipoprotein(a) levels of $40 \%$ and $51 \%$, respectively, whereas there were no significant changes in levels of low-density lipoprotein cholesterol (an increase of 3\% and a decrease of $2 \%$, respectively). We have no data from our trial and know of no other studies that address the speculation raised by Serati et al. as to whether tibolone might activate HPV.

\section{Steven R. Cummings, M.D.}

San Francisco Coordinating Center

San Francisco, CA 94107

Victoria Stathopolous, Ph.D.

Organon

Roseland, NJ 07068

1. Gallagher JC, Baylink DJ, Freeman R, McClung M. Prevention of bone loss with tibolone in postmenopausal women: results of two randomized, double-blind, placebo-controlled, dosefinding studies. J Clin Endocrinol Metab 2001;86:4717-26. 